\title{
The Operation of Coated Tungsten-Based Dispenser Cathodes in Nonideal Vacuum
}

\author{
CHRISTIE R. K. MARRIAN, MEMBER, IEEE, AND ARNOLD SHIH
}

\begin{abstract}
A significant amount of residual gas is always liable to be present in any vacuum system. As a result, the performance of a tungsten-based dispenser cathode may be degraded through interaction with the vacuum ambient. Such a cathode is most sensitive to the oxidizing gases both when dormant and at its operating temperature. In a demountable vacuum system, water vapor and oxygen will almost certainly be the most abundant cathode poisoning agents. The superior emission performance of the coated tungsten dispenser cathodes, e.g. " $M$ " types, makes them desirable alternatives to the uncoated dispenser cathodes such as the " $B$ " or " $S$ " types. It is therefore of interest to study the tolerance of such coated cathodes to imperfect vacuum ambients. This paper describes the behavior of iridium- and osmium-coated dispenser cathodes in background pressures of oxygen and water vapor. Poisoning is predominantly chemical in nature and occurs at significantly lower pressures than would be expected for physical or sputtering damage to the cathode surface. Measurements are presented to determine the maximum background pressure in which the cathodes can maintain their full emission capability. The osmiumcoated cathode has proved to have greater resistance to poisoning than the iridium-coated cathode both at room and operating temperatures. The coated cathodes can operate in about three times as great a pressure of water vapor as oxygen. The results suggest ways that these cathodes can be optimized for operation in nonideal vacuum and that it should be possible to design an osmium-coated cathode that will be able to emit fully at $1310 \mathrm{~K}$ in up to about $5 \times 10^{-8}$ torr of water vapor.
\end{abstract}

\section{INTRODUCTION}

$S^{\prime}$ INCE its development [1], the " $M$ " or coated tungsten-based cathode has been widely used because of its superior emission capability compared to that of the standard " $B$ " or " $S$ " type uncoated dispenser cathodes. Various refractory metal coatings have been investigated [2] of which osmium and iridium [3] have been found to be most successful. More recently, refractory metal alloy overlayers [4], [5] have been shown to give even greater emission enhancement. However, the majority of the characterization of this type of cathode both at this laboratory [6] and elsewhere [7]-[10] has concentrated on the surface chemistry and thermionic physics in ultrahighvacuum (UHV) conditions. While these results may well be applicable to a cathode in the hard vacuum of a well baked sealed-off vacuum tube, there are many applications, some very recent, where a cathode can be expected to face nonideal vacuum conditions. For example, not every demountable system can be baked and pumped as

Manuscript received July 6, 1988; revised September 12, 1988.

The authors are with The Naval Research Laboratory, Washington, DC 20375 .

IEEE Log Number 8824787 thoroughly as a sealed-off tube and the vacuum ambient will be worse as a result. So a cathode in such a system, a free-electron laser injector for example, will have to operate in a potentially hostile environment.

Previous work at this laboratory [11]-[13] has concentrated on chemical poisoning rather than physical sputtering of the cathode [14], [15]. The background pressures at which chemical poisoning occurs are much lower and consequently more important in many applications. We have identified the oxidizing gases as the most severe chemical poisoning gases of which oxygen is the most severe but water vapor is probably the more important as it is more prevalent in a demountable vacuum system. Other studies have also found oxygen to poison an " $\mathrm{M}$ ", cathode at significantly lower pressures than argon [16]. In this paper, we discuss the performance of these " $M$ ", type cathodes in background pressures of these gases and compare the results with those from uncoated dispenser cathodes [11] as well as other published data [16].

Section II briefly describes the experimentation as full details have been published previously [11]-[13]. Osmium- and iridium-coated cathodes are compared in Section III in terms of their emission capability in background pressures of water vapor and oxygen. These results suggest that the osmium-coated cathode is more resistant to poisoning, i.e., can withstand higher pressures, than the iridium cathode. In Section IV, the results from operation in nonideal vacuum are correlated with room-temperature poisoning measurements. Section V describes results from poisoning experiments performed at elevated temperatures, which allows comparison with data from uncoated cathodes. The relative performance of " $\mathrm{B}$,", " $S$," and coated cathodes is further discussed in Section VI. The impregnant composition is shown to play a greater role than the composition of the refractory metal at the surface. Finally conclusions are drawn with respect to the partial pressures of oxygen and water vapor that osmiumcoated dispenser cathodes can withstand for unpoisoned operation.

\section{EXPERIMENTATION}

These measurements were performed in a stainless-steel UHV vacuum system, which was pumped by a $300-1 / \mathrm{s}$ ion and a 170-1/s turbomolecular pump. The base pressure was below $10^{-10}$ torr with the ion pump and below $10^{-9}$ torr with the turbo pump. The system was equipped with a commercial Auger Electron Spectroscopy (AES) 
TABLE I

Cathode Properties

\begin{tabular}{|c|c|c|c|c|}
\hline $\begin{array}{c}\text { Cathode } \\
\text { Coating } \\
\text { Os sputtered } \\
\text { " " aged }\end{array}$ & $\begin{array}{l}\text { Ageing time } \\
\text { hrs }{ }^{\sim} \sim 1485 \mathrm{~K} \\
160\end{array}$ & $\begin{array}{l}\text { Impregnant } \\
\text { Type } \\
411 \\
"\end{array}$ & $\begin{array}{c}\text { Coating/Tungsten } \\
\text { Atomic Ratio } \\
1.4 \\
1.0\end{array}$ & $\begin{array}{c}\text { Effective } \phi^{\star} \\
\text { eV (1) } 1300 \mathrm{~K} \\
1.84 \\
1.83\end{array}$ \\
\hline $\begin{array}{l}\text { Os OMCVD } \\
" \quad " \quad \text { aged }\end{array}$ & 16 & 411 & $\begin{array}{l}1.6 \\
1.1\end{array}$ & $\begin{array}{l}1.87 \\
1.85\end{array}$ \\
\hline Os sputtered & & 532 & 1. 3 & 1.86 \\
\hline $\begin{array}{l}\text { Ir sputtered } \\
" \text { aged }\end{array}$ & 16 & 411 & $\begin{array}{l}1.8 \\
1.1\end{array}$ & $\begin{array}{l}1.90 \\
1.87\end{array}$ \\
\hline
\end{tabular}

system, which was used to characterize the surface elemental composition of the cathodes. There were no indications that the cathodes discussed here were anything but typical of their type.

The osmium-coated cathodes were obtained commercially with the exception of the organo-metallic chemical vapor deposited (OMCVD) sample that was a commercially obtained " $\mathrm{S}$ " cathode coated with $0.4 \mu \mathrm{m}$ of osmium in house [17]. This technique was first used for coating cathodes at the Naval Research Laboratory and produced some very promising results in terms of coating uniformity and emission levels. The iridium-coated cathodes were also commercially obtained " $S$ " cathodes sputtered coated in house with a nominal $1-\mu \mathrm{m}$ layer. The impregnant compositions of the cathodes are represented by three numbers referring to the molar composition of barium, calcium, and aluminum oxides. Thus, the " $S$ " cathode is impregnated with a 411 barium calcium aluminate.

Prior to poisoning measurements each cathode was activated and allowed to equilibrate over at least $16 \mathrm{~h}$ at $\sim 1090^{\circ} \mathrm{C}$ brightness. Subsequently each cathode was evaluated by emission and AES measurements and found to be good examples of their type. The salient properties of the various cathodes described here are listed in Table I. In the text the cathodes are referred to by their coating metal, impregnant composition, and age. The AES measurements have been converted to elemental percent by using measured peak-to-peak heights and published sensitivity factors [18] and literature values for the particular elements and compounds present on these cathodes [19], [20].

Thermionic emission was monitored under low duty cycle $(<0.01$ percent $)$ pulsed $(15 \mu \mathrm{s})$ conditions in a close space diode (CSD) configuration. The anode was cleaned with electron bombardment heating prior to any measurements. No attempt was made to record zero field emission levels. The nature of the experiment necessitated the monitoring of emission when a voltage sufficient to overcome space charge was applied to the anode. The current measured under these conditions was converted to effective work function values by substitution into the
Richardson-Dushman equation with an $A$ value of 120 $\mathrm{A} / \mathrm{cm}^{2}$. Values of these effective work functions are tabulated in Table I for the cathodes in their active states. The work function values are estimated to have an absolute accuracy of $\pm 0.03 \mathrm{eV}$ as a result of alignment uncertainties with the individual cathodes. However, for each cathode the work function values proved reproducible to $\pm 0.015 \mathrm{eV}$ and no systematic degradation was observed during the course of the poisoning experiments. Cathode temperature was monitored with thermocouples mounted close to the emitting surface. The thermocouples were calibrated with a $0.65-\mu \mathrm{m}$ disappearing filament pyrometer. The thermocouple was incorporated in a feedback circuit to maintain reproducible heating profiles and constant cathode temperatures.

The gases used were either research grade purity oxygen or water vapor evaporated from a glass flask of deionized water. The gases were bled into the chamber through UHV leak valves. The poisoning exposures were performed dynamically, i.e., while the chamber was being pumped with the turbomolecular pump. As a result exposure impurities, monitored with a quadrupole mass spectrometer, were less than 0.1 percent. After an exposure, the chamber was restored to UHV by closing the leak valve and evacuating the chamber with both the turbomolecular and ion pumps: this typically took a few seconds. Pressures were measured with a commercial ion gauge and digital controller and are quoted in torr $\mathrm{N}_{2}$ equivalent. Exposures are given in Langmuirs ( $\mathrm{L}$ ) where $1 \mathrm{~L}$ is $10^{-6}$ torr $\cdot \mathrm{s}$.

\section{Operation in Background Pressures of OXYGEN AND WATER VAPOR}

Measurements were made to ascertain the maximum pressure that a given cathode could withstand for unpoisoned (i.e. full emission) operation. Ideally this type of measurement should be performed in an open triode-type structure so that the pressure and hence poisoning flux at the cathode surface can be measured. In a close space diode, the anode-cathode volume is pumped by cathode evaporant so that the poisoning flux will be less than that indicated by a pressure reading remote from the diode. 


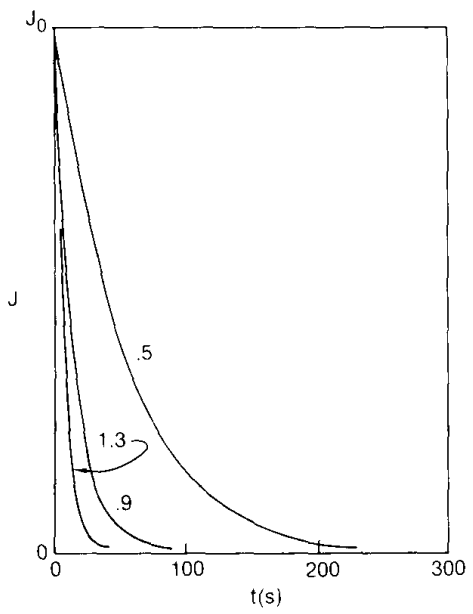

Fig. 1. Time evolution of cathode current showing poisoning in a close spaced diode for three different diode spacings (given in millimeters).

This is illustrated in Fig. 1, where the degradation with time of the cathode emission is plotted for different diode spacings in the same background pressure of $3.3 \times 10^{-8}$ torr of oxygen. As the diode spacing is increased the cathode poisons, i.e., emission falls, more rapidly indicating an increased poisoning flux. The largest spacing shown $(1.3 \mathrm{~mm})$ is the greatest at which effects of space charge can be overcome with our pulser voltage capability ( 2 $\mathrm{kV}$ ). All subsequent measurements described in this section were performed with this diode spacing. It is difficult to be certain that this diode spacing is sufficient to eliminate the poisoning flux reduction. However, measurements at lower current levels and wider spacings suggest that the residual pumping effects is low ( $<20$ percent) at a spacing of $1.3 \mathrm{~mm}$. In addition these measurements compare well with those made using a technique where the poisoning exposure is not made in a close space diode (Section V).

Results for an aged 411 iridium cathode in three different background pressures of oxygen are shown in Fig. 2. Clearly the cathode is extremely sensitive to oxygen poisoning as appreciable poisoning occurs even at $10^{-9}$ torr of oxygen. Two further points can be made from the figure. First, it is very difficult to characterize the results in terms of the steady-state current in a given background pressure. Even after $500 \mathrm{~s}$, there is no sign of stabilization in the cathode current. Operation for longer times is unreliable for experimentation reasons as anode effects on cathode surface composition and temperature become increasingly important. Second, one cannot characterize the results in terms of emission degradation for a given exposure in $\mathrm{L}$. For example, $0.5 \mathrm{~L}$ at $10^{-8}$ torr is sufficient to reduce the cathode current $J$ to $<5$ percent of $J_{0}$, the active state emission level, whereas at $10^{-9}$ torr, the same exposure reduces $J$ to only 60 percent of $J_{0}$. This indicates that the poisoning mechanism is dynamic in nature as opposed to static. Thus, it is the poisoning flux rather than the total exposure that determines the rate of degradation in the cathode emission.

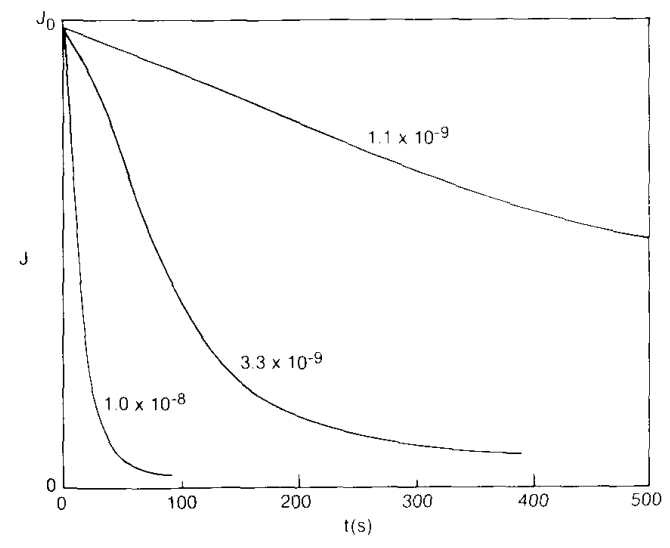

Fig. 2. Poisoning of an aged iridium coated 411 cathode at $1310 \mathrm{~K}$ in different pressures of oxygen (given in torr).

Corresponding results for an aged 411 OMCVD osmium cathode, at the same cathode temperature, are shown in Fig. 3 for three different pressures of oxygen. That the osmium cathode can withstand higher pressures than the iridium cathode is immediately apparent. Again both the difficulty of measuring the steady-state current in a given background pressure and the dynamic nature of the poisoning process are evident.

Other measurements, not shown here, indicate that each cathode type can withstand significantly higher pressures of water vapor than oxygen. For a given temperature, each cathode could tolerate a pressure of water vapor approximately three times that of oxygen. Not unexpectedly there is a strong temperature dependence of these poisoning measurements. In a given background pressure, the current (scaled to $J_{0}$ ) falls more rapidly at lower cathode temperatures.

As mentioned above it is difficult to quantify the data shown in Figs. 2 and 3. Neither emission degradation for a given exposure or steady-state "poisoned" current levels are meaningful. Instead, estimates were made of the highest pressure that the cathode could withstand for full unpoisoned emission. The cathode current was considered unpoisoned if the current did not drop by more than 1 percent in $20 \mathrm{~s}$. Although somewhat arbitrary, this definition of unpoisoned operation enabled realistic comparisons of the different cathodes to be made. A series of measurements were made as a function of cathode temperature and gas type where the background pressure was slowly increased in discrete amounts until the onset of poisoning was observed. These are summarized in Fig. 4, which is an Arrhenius-type plot of pressure (on a logarithmic scale) versus cathode operating temperature (on a $1 / T$ scale). Measurements were possible over a range of temperatures for the osmium cathode, but only one measurement, $4 \times 10^{-9}$ torr water vapor at $1310 \mathrm{~K}$, for the iridium cathode. At lower temperatures the pressure was too low ( $<10^{-9}$ torr) to be measured because of the background pressure in the system. It was not possible to go to higher cathode temperatures as the effects of space 


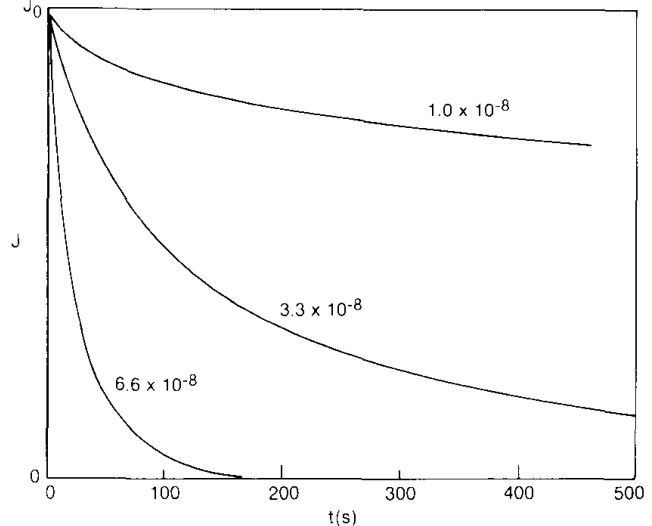

Fig. 3. Poisoning of an aged OMCVD osmium coated 411 cathode at 1310 $\mathrm{K}$ in different pressures of oxygen (given in torr).

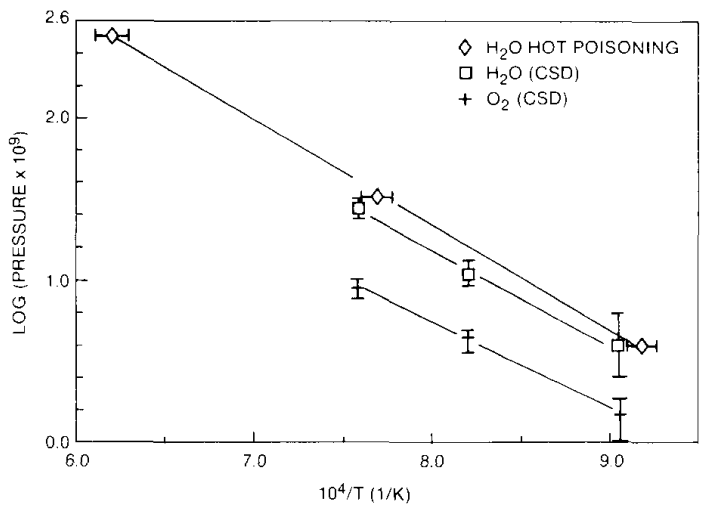

Fig. 4. Arrhenius-type plot showing the temperature dependence of the maximum pressure of water vapour at which full emission can be obtained from coated cathodes. Data from diode measurements are compared with elevated temperature poisoning measurements (Section V).

charge on the cathode emission became important. The third line extending over the full temperature range of the abscissa was obtained from the measurements of poisoning at elevated temperature described in Section V. Good agreement between the two estimates for the pressure tolerance of 411 osmium cathodes in water vapor is apparent. Although there are few points in Fig. 4, the indication is that they lie on straight parallel lines. This suggests a temperature dependance of the resistance to the poisoning flux of the form of an Arrhenius rate equation. In other words, the maximum pressure the cathodes can withstand is proportional to:

$$
\exp \left(-E_{A} / k T\right)
$$

where $k$ is Boltzmann's constant and $T$ is the cathode temperature. This gives values for $E_{A}$, which can be thought of as an effective activation energy, of about $1.2 \pm .1$ $\mathrm{eV}$. This value is somewhat lower than that estimated for an " $S$ " cathode [11] from a smaller data sample. Data (not shown here) from 532 ' $M$ ' cathodes suggest rather higher values for $E_{A}$ as noted with a "B" cathode. A higher value was also noted in studies of a 532 osmium- coated cathode [16]. Identification of the physical processes responsible for these differences is the subject of continuiing work at this laboratory.

\section{Reactivation Following Room-Temperature EXPOSURES}

This type of measurement has been extensively reported previously [11]-[13] by this laboratory. The cathode is first activated and allowed to cool to room temperature. It is then exposed to a specific amount of gas (in this case $10 \mathrm{~L}$ ) before being reactivated in a close space diode under UHV conditions. The severity of the poisoning is characterized by the delay in the cathode emission once the cathode has reached the reactivation temperature. The delay is defined as the time required to reach 37 percent of the active state current, which allows comparison with results for uncoated tungsten dispenser cathodes in [11].

Results for the 411 coated cathodes are shown in Fig. 5, which is an Arrhenius-type plot of reactivation delay against reactivation temperature. Shown are results from iridium- and OMCVD osmium-coated cathodes following exposure at room temperature to $10 \mathrm{~L}$ of water vapor. In terms of turn-on delay, the iridium cathode is clearly inferior. The two data sets for the osmium cathode reflect different ages of the cathode characterized by the osmiumto-tungsten atomic ratio observed with AES at the cathode surface. Unlike the iridium cathode, the osmium cathode shows a dependance of reactivation delay with surface composition, the osmium-rich cathode having the shorter delay for a given reactivation temperature. The same variation with surface composition was observed with a sputter-coated osmium cathode. As previously reported [17], the OMCVD cathode surface composition changes more rapidly in early life than sputter coated cathodes. As a result, the aging required for the same surface compositional change is considerably different for the two cathodes. Aging times and temperatures are given in Table I. However, no such variation was seen with an osmiumcoated 532 cathode or the iridium-coated 411 cathode. Thus, the lower reactivation delay seems to be a property of 411 cathodes with an osmium-rich surface. Such an osmium-rich cathode has a slightly higher work function than the 50-percent osmium cathode [17], so it suggests that this type of cathode can be optimized for turn-on delay at the expense of a slight reduction in emission.

No similar dependance on surface composition was observed with any coated cathodes with oxygen poisoning; see Fig. 6. Here, data from each cathode type fall along the same straight line irrespective of surface composition, Also apparent from the figure is the greater poisoning sensitivity of both cathode types to oxygen. Thus, there seems to be a correlation between the type of measurements described here and in the previous section. For example, when comparing two cathodes, the one that has the shorter turn-on delay following room temperature poisoning will be able to withstand a higher background pressure of the poisoning gas while operating. 


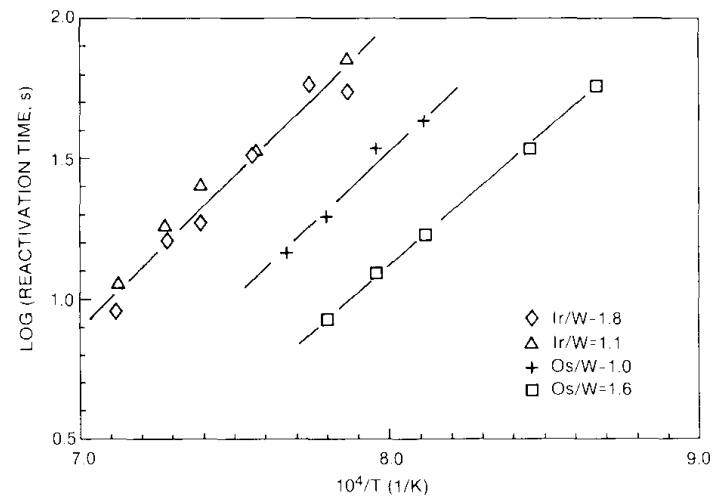

Fig. 5. Arrhenius-type plot showing the temperature dependence of the reactivation delay of osmium- and iridium-coated 411 cathodes follow. ing exposure to water vapour at room temperature.

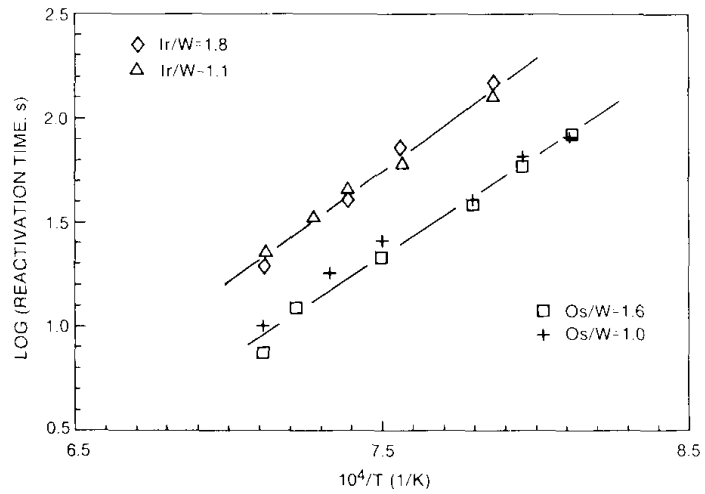

Fig. 6. Arrhenius-type plot showing the temperature dependence of the reactivation delay of coated 411 cathodes following exposure to oxygen at room temperature.

The explanation for the reduction in water vapor poisoning with osmium-rich surfaces is not clear. AES revealed no significant differences in the different cathode surfaces after exposure. In each case, the oxygen content of the surface increased and the low-energy barium peaks indicated further oxidation of the barium oxide on the active cathode surface. It seems that there is some subtle change in the binding energy of the absorbed water vapor that results in differing desorption products once the cathode is heated. AES is probably insensitive to this type of difference, which may, however, be detectable with a technique such as ultraviolet photoelectron spectroscopy [21].

\section{Poisoning at Elevated Temperatures}

This type of measurement has been described in detail [11] when results from uncoated tungsten-based dispenser cathodes were described. Essentially the cathode is exposed to water vapor at an elevated temperature and then allowed to cool to room temperature. The turn-on delay during the subsequent reactivation in UHV is used to monitor the cathode surface following the exposure at the elevated poisoning temperature. Thus, if the turn-on delay is zero $(<1 \mathrm{~s}$ in our experimentation), the cathode

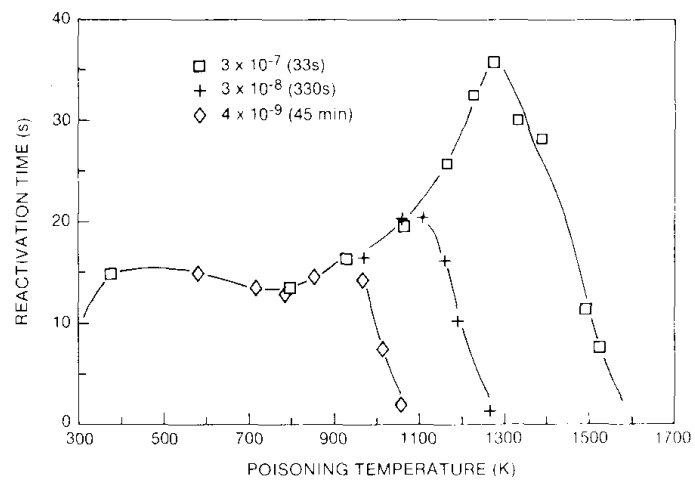

Fig. 7. Reactivation delay plotted as a function of poisoning temperature for an aged osmium-coated 411 cathode exposed to $10 \mathrm{~L}$ of water vapor at three different pressures. Extrapolations to the break-even temperature provide the data plotted in Fig. 4

was considered not to be poisoned by the exposure. Total exposures were kept constant $(10 \mathrm{~L})$ although the poisoning pressure (and hence poisoning time) were varied. Results for a commercially obtained sputter-coated M411 cathode are shown in Fig. 7. Here, the reactivation delay is plotted against the temperature at which the cathode was exposed to water vapor. In each case the reactivation was measured at a fixed temperature of $1270 \mathrm{~K}$.

Similar features are apparent as in results from uncoated cathodes [11]. As the poisoning temperature is increased above room temperature, the reactivation delay increases probably as a result of an increase in the surface reactivity. After a short plateau region the reactivation delay increases again although not as dramatically as observed with the uncoated cathodes. This was attributed to diffusion of water vapor below the surface so it appears that the presence of the " $M$ " coating is slowing the diffusion process. At still higher poisoning temperatures the reactivation delay drops as the cathode begins to be able to reactivate as fast as it poisons.

By extrapolation one can estimate the "break even" temperature at which the cathode can operate without poisoning in the pressure used during the poisoning exposure. Clearly these values of pressure and break even temperature should correlate with the close space diode measurements of the highest pressure for unpoisoned operation described in Section III. Fig. 7 shows the results with three different poisoning pressures, which are plotted versus the corresponding break even temperatures in Fig. 4. The pressure range is the maximum possible in our vacuum system with manual control of the various leak and gate valves. At higher pressures the exposure time is too short, at lower pressures the vacuum ambient becomes an important fraction of the poisoning pressure. In view of the expected uncertainties in the two methods of measuring the pressure for unpoisoned operation, agreement is good; see Fig. 4. The main uncertainty with the diode measurements is the flux at the cathode surface. Whereas this is known in the hot poisoning measurements, the extrapolation to "zero" reactivation delay is subject to er- 


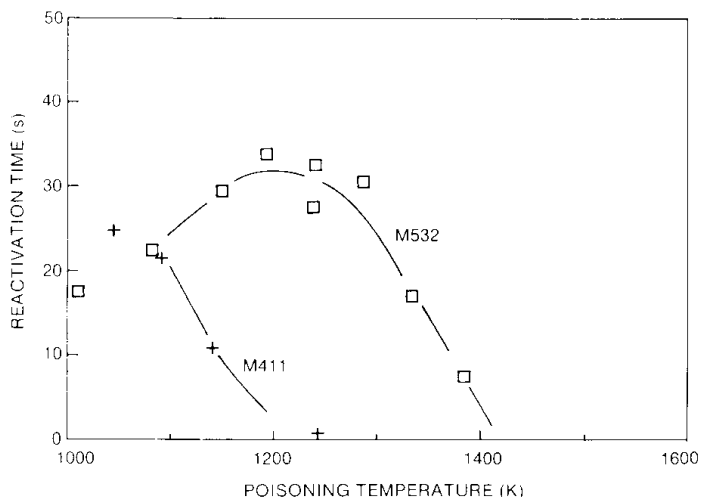

Fig. 8. Comparison of osmium coated cathodes with 411 and 532 impregnants. The reactivation delay is plotted as a function of the temperature at which the cathodes were exposed to $10 \mathrm{~L}$ of water vapor.

ror. In addition it should be born in mind that the measurements were made on two different examples of the same cathode type, although they were similar in terms of measured work function and surface composition.

As shown for the uncoated cathodes, the barium-rich impregnant allows the osmium M411 cathode to withstand higher water vapor pressures than the osmium M532 cathode; see Fig. 8. At lower poisoning temperatures the behavior is, within experimental error, identical. For clarity, only the higher poisoning temperatures are shown. It is instructive to compare this figure with Fig. 7 from [11] where the "B," i.e., B532, and the "S," i.e., S411, cathodes are compared. Within the accuracy expected from the extrapolation the break-even temperatures seem dependent on the impregnant composition rather than the cathode surface coating, i.e., for the 411 cathodes 1210 $\pm 40 \mathrm{~K}$ and for the 532 cathodes $1430 \pm 30 \mathrm{~K}$. Further comparisons between coated and uncoated cathodes are made in the next section.

\section{Discussion}

\section{A. Comparison of Coated and Uncoated Cathodes}

Initial poisoning measurements on these types of cathodes found that a M532 cathode poisoned more severely than an uncoated cathode with a 311 impregnant [12]. Subsequently [13], a strong dependence of the reactivation delay following poisoning was found on the barium content of the cathode impregnant. This is also apparent in comparisons of this work with more recent results [11]. Fig. 9 shows a summary of reactivation delays following exposure to water vapor of the various cathodes discussed here and in [11]. The measurements reflect the time that the cathode current takes to recover to 37 percent of its active state value after exposure at room temperature to $10 \mathrm{~L}$ of water vapor. Each line represents a least squares regression through data from at least two cathodes of each type. The two lines for the M411 cathodes correspond to two different surface osmium to tungsten ratios. No similar variation was observed with the M532 cathodes. The error bars represent variation between the individual cathodes of each type.

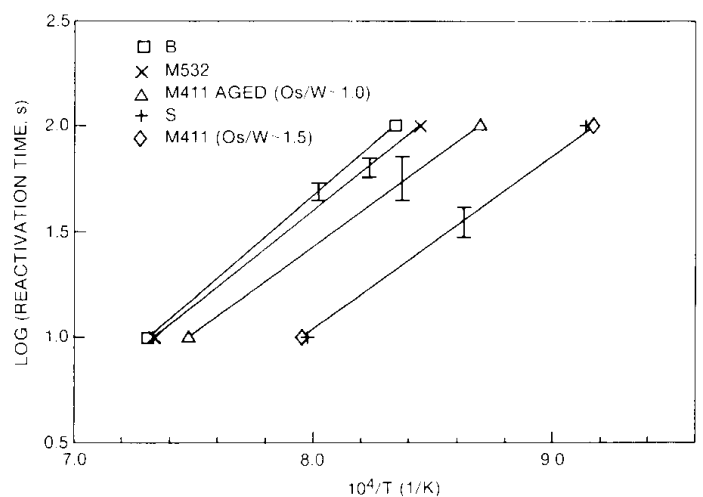

Fig. 9. Arrhenius-type plot showing the temperature dependence of the reactivation delay of coated and uncoated cathodes following room-temperature exposure to water vapor.

Two points stand out. First, the impregnant composition plays a more important role than the cathode coating. The M532 and " $B$ "' cathodes are almost identical and the " S" and osmium-rich M411 cathodes fall along the same line. Second, the slopes of all the lines are similar. Expressing the slope in terms of an activation energy $E_{A}$, they all correspond to a value of $E_{A}$ between 1.7 and 2.0 $\mathrm{eV}$.

The first point must be qualified by the observation that for the M411 cathodes the reactivation delay was observed to increase as the cathodes were aged and the surface osmium concentration decreased. As mentioned above, this effect is peculiar to water vapor and was not observed with oxygen poisoning.

It should be emphasized that the poisoning and reactivation processes in these types of cathodes is extremely complicated. As a result it is not possible to develop a (relatively) simple model to describe quantitatively the various results presented here and elsewhere. However, it does appear that the impregnant composition plays a more important role in determining the reactivation delay than the surface coating. Effects due to surface coating are apparent with M411 cathodes, where reduced reactivation delays (and higher tolerance of background pressure) can be obtained by maintaining an osmium-rich surface. Although this results in a slight emission degradation (compared to a regular " $M$ " cathode), it is an example of how a cathode can be optimized for operation in a nonideal vacuum.

\section{B. Pressure Tolerance of ' $M$ ' Cathodes}

The results presented here indicate that, for operation in nonideal vacuum, osmium-coated cathodes with 411 barium calcium aluminate impregnant are to be preferred. At near $1310 \mathrm{~K}$, such a cathode can provide its full emission capability in up to about $3 \times 10^{-8}$ torr of water vapor or about $1 \times 10^{-8}$ torr of oxygen. However, if such a cathode can be designed to maintain an osmium-rich surface (osmium-to-tungsten atomic ratio $>1.5$ ), these pressures can be exceeded. Preliminary measurements indicate that the osmium-rich cathode poisons, in a given 
pressure of water vapor, at the same rate as a regular " $\mathrm{M}$ ", cathode when at about $40 \mathrm{~K}$ lower in temperature. This suggests that a coated cathode with an osmium-to-tungsten ratio of $\sim 1.6$ with a 411 impregnant could provide its full emission at $1310 \mathrm{~K}$ in close to $5 \times 10^{-8}$ torr of water vapor.

With the use of conventional dispenser cathode fabrication techniques, it may well not be possible to design a cathode for operation in significantly greater background pressures. The key appears to be the increased dispensing of barium uniformly over the cathode surface. Alternate cathode fabrication strategies such as the Controlled Porosity Dispenser cathode [22] or recent improvements on the reservoir or " $L$ " cathode [23] may well provide greater tolerance of nonideal vacuum.

\section{Conclusions}

Detailed studies of the poisoning and reactivation kinetics of coated tungsten-based dispenser cathodes indicate that they can be optimized for tolerance of imperfect vacuum conditions. As with uncoated cathodes, the 411 impregnant allows undegraded emission operation in higher background pressures than the 532 impregnant. Our results suggest that osmium coatings are superior to iridium coatings for these applications. In conditions where water vapor is the predominant chemical poisoning gas, a coated with an osmium-to-tungsten ratio of $\sim 1.5$ with a 411 impregnant was found to have a poisoning tolerance similar to an " $\mathrm{S}$ " cathode.

The poisoning kinetics of dispenser cathodes at operating temperatures are dynamic in nature. The degradation in emission at a given temperature is characterized by the poisoning flux, i.e., the background gas pressure, as well as the total exposure. Thus, one cannot state that a given exposure results in a given decrease in cathode current.

The poisoning and reactivation mechanisms appear to be similar for coated and uncoated cathodes. However, the " $M$ " coating prevents some of the in-diffusion that causes a reduction in the increase in poisoning at elevated temperatures. In addition, the interaction with the poisoning gas is affected to some extent by the surface refractory metal composition. Although with oxygen, the interaction is not dependent on the tungsten-coating metal ratio, osmium-rich coated cathodes poison less severely with water vapor. This allows the possibility of optimizing the coating composition for operation in residual pressures of water vapor at the expense of a slight reduction in cathode emission.

\section{ACKNOWLEDGMENT}

The authors would like to express their appreciation for the technical assistance of $\mathrm{C}$. Hor.

\section{REFERENCES}

[1] P. Zalm and A. J. A. van Stratum, Philips Tech. Rev., vol. 27, p. 69. 1966.

[2] G. K. Bhide and F. E. Wray, J. Phys. D, vol. 3. p. 443, 1970

[3] T. Pankey and R. E. Thomas, Appl. Surface Sci., vol. 8, p. 50, 1981.
14] M. C. Green, H. B. Skinner, and R. A. Tuck, Appl. Surface Sci., vol. 8 , p. 13,1981 .

[5] G. A. Haas, A. Shih, and C. R. K. Marrian, Appl. Surface Sci., vol. 24, p. $447,1985$.

[6] C. R. K. Marrian, A. Shih, and G. A. Haas, Appl. Surface Sci, vol. 16, p. $1,1983$.

[7] K. D. Rachocki, B. C. Lamartine, and T. W. Haas, Appl. Surface Sci., vol., 16, p. 40, 1983

[8] J. Hasker and N. C. J. van Hijningen, Appl. Surface Sci., vol. 24, p. $318,1985$.

[9] R. Forman, Appl. Surface Sci., vol. 24, p. 587, 1985

[10] R. Forman, Appl. Surface Sci., vol. 29, p. 127, 1987.

[11] C. R. K. Marrian and A. Shih, IEEE Trans. Electron Devices, vol. ED-33, p. 1874 , Nov. 1986

[12] C. R. K. Marrian, G. A. Haas, and A. Shih, Appl. Surface Sci., vol. 16, p. 73,1983

[13] - Appl. Surface Sci., vol. 24, p. 391, 1985.

[14] R. A. Tuck, Appl. Surface Sci., vol. 2, p. 128, 1979.

[15] D. J. Schipper and M. H. G. Steeghs, Appl. Surface Sci., vol. 24, p. 291,1985

[16] J. van de Berg and P. R. Boelens, in Residual Gases in Electron Tubes. T. A. Giorgi and P. della Porta, Eds. London: Academic, 1972, p. 99.

[17] A. Shih, A. Berry, C. R. K. Marrian, and G. A. Haas, IEEE Trans. Electron Devices, vol. ED-34, pt. 2, p. 1193, May 1987.

[18] L. E. Davis, N. C. MacDonald, P. W. Palmberg, G. E. Riach. and R. E. Weber, Handbook of Auger Spectroscopy, 2nd ed., Physical Electronics Industries, Inc., 1976.

[19] D. Brion, J. C. Tonnerre, and A. Schroff, Appl. Surface Sci., vol. 16, p. 55,1983

[20] A. Shih, C. Hor, and G. A. Haas, Appl. Surface Sci., vol. 2, p. 112, 1979 .

[21] D. Mueller, A. Shih, E. Roman, T. Madey, R. Kurtz, and R. Stockbauer, "Interaction of $\mathrm{O}_{2}, \mathrm{CO}_{2}$ and $\mathrm{H}_{2} \mathrm{O}$ with model dispenser cathodes," presented at the 1988 Tri-Service Cathode Workshop. Asbury Park, NJ, Mar. 1988.

[22] R. E. Thomas, and T. Pankey, Jr., "Controlled-porosity dispenser cathode," U.S. Patent 4101800 , July 18, 1978

[23] H. J. Lemmens, M. J. Jansen, R. Loosjes, Philips Tech. Rev., vol. 11, p. $341,1950$.

*

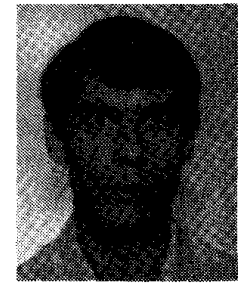

Christie R. K. Marrian (M 88 ) received the B. A. and Ph.D. degrees from the Electrical Engineering Department of the University of Cambridge in 1973 and 1978 , respectively.

Following a postdoctral fellowship at C.E.R.N. in Geneva, Switzerland, he came to the Naval Research Laboratory in 1980 . He joined the Surface Physics branch and has worked extensively on high current density thermionic emitters and their degradation when exposed to vacuum ambient gases. His current research interests include the operation of dispenser cathodes in hostile vacuum environments. $\mathrm{He}$ is also involved in studies of surface modification and patterning on the nanometer scale.

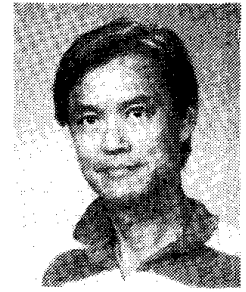

Arnold Shih was born in Shanghai, China. He received the A.B. degree in physics from the University of California at Berkeley in 1965 and the Ph.D. degree in physics from Columbia University, New York, NY, in 1972.

From 1972 to 1975 , he was with the Surface Chemistry Section of the National Bureau of Standards. In 1975, he joined the Naval Research Laboratory, and he is currently serving as Head of the Surface Electronics and Nanometric Physics Section in the Surface Physics Branch. His interest has been mainly in surface analysis with various electron spectroscopies, particularly on the systems related to thermionic cathodes. 